\title{
A PRACTICAL STUDY OF ADAPTIVE FINGER REASSIGNMENT AND REPLACEMENT IN THE SOFT HANDOVER REGION*
}

\author{
S. Choi
}

M.-S. Alouini, K. A. Qaraqe

Texas A\&M Univ. at Qatar

Elec. Eng.

Doha, Qatar

\author{
H.-C. Yang \\ Univ. of Victoria \\ Elec. \& Comp. Eng. \\ BC, Canada
}

Y.-C. Ko

\author{
Korea Univ. \\ Elec. \& Comp. Eng. \\ Seoul, Korea
}

\begin{abstract}
We present the effects of correlated or/and non-identical fading channels as well as outdated/imperfect channel estimations on the performance of newly proposed finger reassignment and replacement schemes for RAKE receivers in the soft handover region. The performance of these schemes were previously analyzed by the authors over independent and identically distributed Rayleigh fading channels. In this paper, we consider the exponentially decaying power delay profile among paths with the exponential and constant correlation models. The effect of outdated/imperfect channel estimations is also evaluated. Simulation results show that our proposed schemes are still offering an interesting performance versus network overhead tradeoff in the practical channel environments considered in this paper.
\end{abstract}

\section{INTRODUCTION}

RAKE reception is a technique which uses several baseband correlators called fingers to individually process multipath signal components. The outputs from the different correlators are coherently combined to improve the signalto-noise ratio (SNR) and to therefore lower the probability of deep fades [1, Section 9.5.1]. Since they rely on resolvable multipaths to operate, RAKE receivers are used in conjunction with wideband systems such as wideband code division multiple access (WCDMA) and ultra wideband (UWB) systems. In the handover (HO) region, the number of available resolvable paths can be quite high since they come from the serving base station (BS) and the target BSs. At the same time, due to the hardware and battery life time constraints for mobile units, RAKE receivers have typically a limited number of fingers. Hence, it is natural to consider how to judiciously select a subset of paths for RAKE reception in the soft $\mathrm{HO}(\mathrm{SHO})$ region

* This is an invited paper for the Adaptive Processing in Wireless Communications Session organized by Dr. M. Ibnkahla. This work was supported in part by the Qatar Foundation (QF) for Education, Science, and Community Development, Qatar, in part by Qatar Telecom (Qtel), Qatar, in part by a Discovery Grant from NSERC, Canada, and in part by the center for Broadband OFDM Mobile Access (BrOMA) at POSTECH through the ITRC program of the Korean MIC, supervised by IITA (IITA-2005-C1090-0502-0008). in order for the receivers to achieve the required performance while (i) maintaining a low complexity and low processing power consumption and (ii) using a minimal amount of additional network resources.

Even though many low-complexity diversity combining schemes have been proposed and studied over the last decade [2-7], they still result in a significant increase in network overhead (known as a SHO overhead [8, Section 9.3.1.4]) if they are used without any modification. Recently, by considering two BSs (one serving and one target BSs) the authors proposed and analyzed the performance of two new finger assignment schemes (namely the Full Generalized Selection Combining (GSC) scheme [9] and the Block Change scheme [10]) that maintain a low complexity and reduce the SHO overhead. The main idea behind these schemes is that a receiver in the SHO region uses the additional resolvable paths from the target BS only if needed. For analytical tractability, $[9,10]$ assumed independent and identically distributed (i.i.d.) Rayleigh fading channels and perfect channel estimation, and as such, these papers were able to offer (i) some closed-form expressions for the statistics of the output SNR and (ii) analytical-based study of the tradeoff among error performance, path estimation load, and SHO overhead. However, there are a number of real-life scenarios in which this i.i.d. assumption is not valid especially in multipath diversity over frequency-selective channels and as such the study on the impact of various realistic fading channels is very important.

In this paper, as an additional complexity measure we evaluate the average number of required SNR comparisons. Then we further look into the schemes proposed in $[9,10]$ in more practical fading environments. More specifically, we consider through various simulations the effects of path unbalance as well as path correlation on the performance. The impact of outdated or imperfect channel estimation is also investigated.

\section{FINGER ASSIGNMENT SCHEMES}

\subsection{Channel and System Model}

Let $\gamma_{i}$ denote the instantaneous received SNR of the $i$ th resolvable path from the serving or target BS. We assume that the signals from each BS experience correlated or/and non-identically distributed Rayleigh fading environments. 
For the non-identical fading channels, we assume an exponentially decaying power delay profile (PDP) among paths and for the correlated fading channels, we consider two channel correlation models : constant and exponential correlation models.

We consider systems that employ RAKE type of reception. We assume that, in the SHO region, there are $L$ available resolvable paths from the serving BS and $L_{a}$ additional available paths from the target BS, and depending on the channel conditions only $L_{c}$ paths among $L$ or $L+L_{a}$ paths are used for RAKE reception according to the mode of operation described in what follows.

\subsection{Mode of Operation}

We assume that the $L_{c}$ out of total $L$ resolvable paths from the serving BS are by default assigned to the RAKE fingers of the mobile unit in the SHO region following $L_{c} / L$ GSC type of combining. Only when the output SNR falls below a predetermined fixed SNR threshold, $\gamma_{T}$, (known also as the target SNR), the receiver asks for the additional resources from the target BS and scans the additional $L_{a}$ resolvable paths from the target BS.

After scanning, with the Full GSC scheme the receiver selects again the strongest $L_{c}$ paths but now among the $L+L_{a}$ available paths (i.e., the receiver uses $L_{c} /\left(L+L_{a}\right)$ GSC). On the other hand, with the Block Change scheme the receiver compares the sum of the SNRs of $L_{a}$ paths from the target BS with the sum of the $L_{a}$ weakest SNRs among the currently used $L_{c}$ paths from the serving BS and selects the better group to form $L_{c}$ paths again. As shown in [10], unlike the Full GSC scheme in [9], the Block Change scheme just compares two sums and as such avoids reconfiguring all fingers and also ends up further reducing the SHO overhead in the SHO region at the expense of a slight performance loss.

\section{AVERAGE NUMBER OF SNR COMPARISONS}

In $[9,10]$, to study the tradeoff between the performance and the complexity, the average number of path estimations was examined as a complexity measure. In this section, we quantify the complexity of the proposed schemes by calculating also the average number of SNR comparisons, denoted by $M$, needed during the SHO process. Noting that $M_{G\left(L_{c}, L\right)}=\sum_{k=1}^{\min \left[L_{c}, L-L_{c}\right]}(L-k)$ for $L_{c} / L$ GSC, we can obtain the expressions for $M$ needed by the proposed schemes as

$$
\begin{aligned}
M_{F}= & \left(1-F_{\Gamma_{L_{c}: L}}\left(\gamma_{T}\right)\right) M_{G\left(L_{c}, L\right)} \\
& +F_{\Gamma_{L_{c}: L}}\left(\gamma_{T}\right) M_{G\left(L_{c}, L+L_{a}\right)}
\end{aligned}
$$

for Full GSC, and

$$
\begin{aligned}
M_{B}= & \left(1-F_{\Gamma_{L_{c}: L}}\left(\gamma_{T}\right)\right) M_{G\left(L_{c}, L\right)} \\
& +F_{\Gamma_{L_{c}: L}}\left(\gamma_{T}\right)\left(M_{G\left(L_{c}, L\right)}+M_{G\left(L_{a}, L_{c}\right)}+1\right)
\end{aligned}
$$

for Block Change where $\Gamma_{i: j}$ is the sum of the $i$ largest SNRs among $j$ ones, i.e., $\Gamma_{i: j}=\sum_{k=1}^{i} \gamma_{k: j}$ where $\gamma_{k: j}$ is the $k$ th order statistics (see [3] for terminology) and as such $F_{\Gamma_{i: j}}\left(\gamma_{T}\right)$ is the well-known CDF of $i / j$-GSC output SNR [11, Eq. (9.440)] evaluated at $\gamma_{T}$.

Fig. 1 shows the average number of SNR comparisons of the Full GSC and the Block Change schemes in comparison with traditional GSC. From the results obtained in $[9,10]$, we know that compared to Full GSC, the Block Change scheme has the advantage of reducing the SHO overhead with a slight performance loss while maintaining the same number of path estimations. However, as we can see from this figure, the Block Change scheme requires less SNR comparisons. This is because, instead of reconfiguring all the fingers as used in the Full GSC scheme, with Block Change scheme, if needed, the receiver just compares the sums of two groups, the sum of the currently connected $L_{a}$ smallest paths from the serving BS and the sum of the $L_{a}$ additional paths from the target BS.

\section{EFFECT OF PATH UNBALANCE/CORRELATION}

In practice, the i.i.d. fading scenario on the diversity paths as assumed in $[9,10]$ is not always realistic due to, for example, the different adjacent multipath routes and the resulting unbalance and correlation among paths. In this section, we assess the effect of non-identically distributed paths with correlation on the performance for both schemes. More specifically, instead of the uniform PDP considered in $[9,10]$, we now consider an exponentially decaying PDP, $\bar{\gamma}_{i}=\bar{\gamma}_{1} e^{-\delta(i-1)}$, where $\bar{\gamma}_{i}$ is the average SNR of $i$-th path out of total available resolvable paths from each $\mathrm{BS}$ and $\delta$ is the average fading power decaying factor. For the correlated paths, we consider constant and exponential correlation models. For the constant correlation model, the same power correlation coefficient, $\rho \in[0,1]$, is assumed between all the path pairs while the exponential correlation model assumes an exponential power correlation coefficient, $\rho^{\left|i-i^{\prime}\right|}$, between any pair of paths, $\gamma_{i}$ and $\gamma_{i^{\prime}}$. Note that $\delta=0$ means identically distributed paths while $\rho=0$ means independent fading paths. Setting $\delta=0$ and $\rho=0$, we revert to the i.i.d. fading channels considered in $[9,10]$.

In Fig. 2, we plot the average bit error rate (BER) of binary shift keying (BPSK) versus $\bar{\gamma}_{1}$ with the Full GSC and the Block Change schemes over an exponentially decaying PDP with (a) an exponential correlation and (b) a constant correlation across the multipaths. In all cases, the Full GSC scheme shows a slightly better performance as observed in the i.i.d. case analyzed in $[9,10]$. These results also show that PDP induces a non-negligible degradation in the performance and therefore must be taken into account for the accurate prediction of the performance of proposed schemes. Moreover, we can observe that constant correlation suffers a minor performance degradation in comparison to exponential correlation. 


\section{EFFECT OF OUTDATED OR IMPERFECT CHANNEL ESTIMATES}

In general, diversity combining techniques rely, to a large extent, on accurate channel estimation. As a typical first step in performance analysis, perfect estimation was assumed in $[9,10]$. However, in practice these estimates must be obtained in the presence of noise and time delay. Hence, the effects of channel estimation error or channel decorrelation on the performance of diversity systems is of interest. In this section, we study the effect of outdated or imperfect channel estimates on the performance. For simplicity, all the diversity paths are assumed to be i.i.d. Let $\gamma^{\tau}$ be the estimated received signal power. Due to imperfect or outdated channel estimates, $\gamma^{\tau}$ may or may not be the same as $\gamma$. Hence, we can assume that $\gamma^{\tau}$ is the correlated sample from $\gamma$ with a power correlation factor, $\rho^{\tau} \in[0,1]$, between $\gamma$ and $\gamma^{\tau}$. Here, $\rho^{\tau}$ can be viewed as a measure of channel fluctuation rate and a measure of the channel estimation quality as well. As an example, from the well-known Clark's model, we know $\rho^{\tau}=J_{0}^{2}\left(2 \pi f_{D} \tau\right)$ [1, Section 2.1.1] where $J_{0}()$ is the zero-order Bessel function of the first kind, $\tau$ is the time delay, and $f_{D}$ is the maximum Doppler frequency shift. Note that $\rho^{\tau}=0$ means completely outdated channel estimates while $\rho^{\tau}=1$ an uptodate and perfect channel estimates.

Fig. 3 compares the effect of the correlation factor, $\rho^{\tau}$, on the average BER of BPSK with the Full GSC and the Block Change schemes for several values of the output threshold, $\gamma_{T}$, over i.i.d. Rayleigh fading channels. We can see from these curves that in all cases the diversity gain offered by the proposed schemes decreases as $\rho^{\tau}$ decreases, as expected. It is very notable that contrary to the analysis over perfect channel estimations, the Block Change scheme shows a lower error probability than the Full GSC scheme when $\rho^{\tau}=0$ and 0.5 for $\gamma_{T}=5$ and 15 $\mathrm{dB}$. Recall that the Block Change scheme compares two sums of paths from the serving and target BSs while the Full GSC scheme relies on the SNR of each path. Therefore, the Block Change scheme is more robust to the channel estimation errors especially when the comparisons of the two sums are needed. In other words, the more often the combined SNR is below the threshold, the less sensitive to the channel estimation error the Block Change scheme is while the more sensitive the Full GSC scheme is.

\section{CONCLUSION}

In this paper, we examined the effects of various practical considerations on the performance of some newly proposed finger assignment schemes for RAKE reception in the SHO region which were previously analyzed over ideal i.i.d fading environments $[9,10]$. We obtained the average number of SNR comparisons as an another complexity measure. Then, through computer simulations, we considered the impact of an exponentially decaying PDP as well as a fading correlation among paths. The ef- fect of outdated or imperfect channel estimations was also evaluated. In summary, with the analytical methods presented in $[9,10]$ and the simulation results presented in this paper, we are providing a general comprehensive framework for the assessment of the proposed finger assignment schemes.

\section{REFERENCES}

[1] G. L. Stüber, Principles of Mobile Communication, 2nd ed. Norwell, MA: Kluwer Academic Publishers, 2001.

[2] M. Z. Win and J. H. Winters, "Analysis of hybrid selection/maximal-ratio combining in Rayleigh fading," IEEE Trans. Commun., vol. 47, no. 12, pp. 1773-1776, Dec. 1999.

[3] M.-S. Alouini and M. K. Simon, "An MGF-based performance analysis of generalized selection combining over Rayleigh fading channels," IEEE Trans. Commun., vol. 48, no. 3, pp. 401-415, Mar. 2000.

[4] A. Annamalai and C. Tellambura, "Analysis of hybrid selection/maximal-ratio diversity combiner with Gaussian errors," IEEE Trans. Wireless Commun., vol. TWC-1, no. 3, pp. 498-512, July 2002.

[5] H.-C. Yang, "New results on ordered statistics and analysis of minimum-selection generalized selection combining (GSC)," IEEE Trans. Wireless Commun., vol. 5, no. 7, pp. 1876-1885, July 2006.

[6] M.-S. Alouini and H.-C. Yang, "Minimum estimation and combining generalized selection combining (MEC-GSC)," in Proc. IEEE Int. Symp. on Information Theory (ISIT'05), Adelaide, Australia, Sept. 2005.

[7] H.-C. Yang and M.-S. Alouini, "MRC and GSC diversity combining with an output theshold," IEEE Trans. Veh. Technol., vol. 54, no. 3, pp. 1081-1090, May 2005.

[8] H. Holma and A. Toskala, WCDMA for UMTS, revised ed. New York, NY: John Wiley \& Sons, 2001.

[9] S. Choi, M.-S. Alouini, K. A. Qaraqe, and H.-C. Yang, "Performance analysis of RAKE receivers with finger reassignment," in Proc. IEEE Int. Conf. on Commun. Systems (ICCS'06), Singapore, Nov. 2006.

[10] _ , "Soft handover overhead reduction by RAKE reception with finger replacement," in Proc. IEEE Int. Conf. on Commun. Systems (ICCS'06), Singapore, Nov. 2006.

[11] M. K. Simon and M.-S. Alouini, Digital Communication over Fading Channels, 2nd ed. New York, NY: John Wiley \& Sons, 2005. 


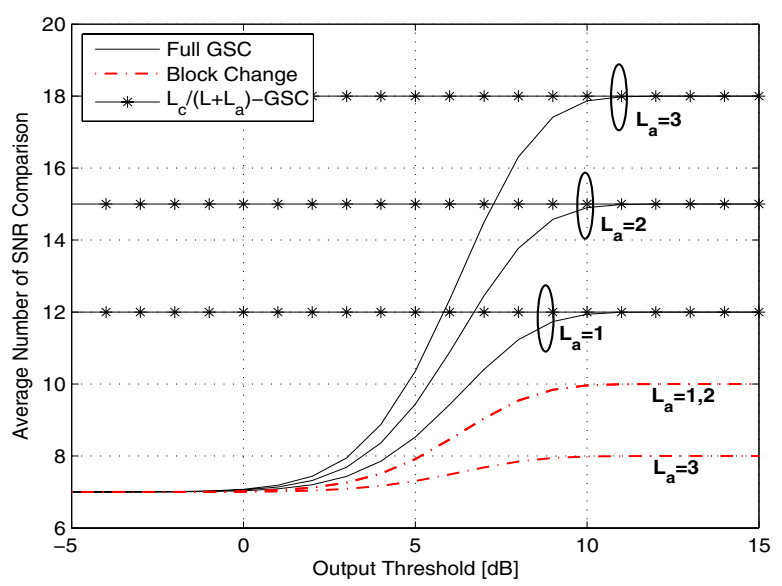

Fig. 1. Average number of SNR comparison versus the output threshold, $\gamma_{T}$, for the Full GSC and the Block Change schemes over i.i.d. Rayleigh fading channels when $L=5, L_{c}=3$, and $\bar{\gamma}=0 \mathrm{~dB}$.

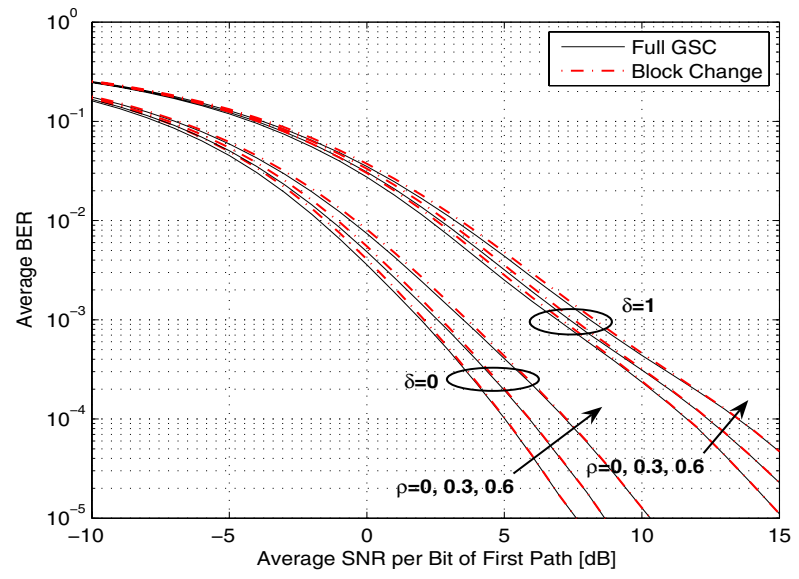

(a) Exponential Correlation

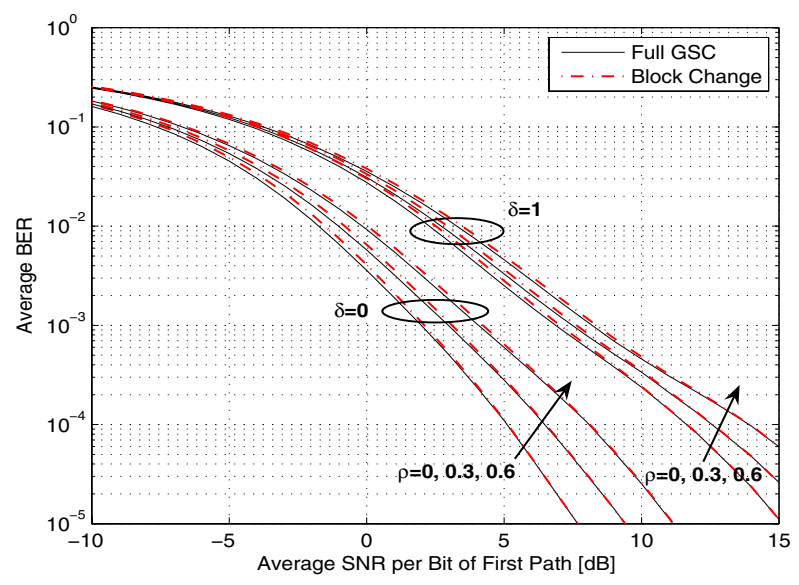

(b) Constant Correlation

Fig. 2. Average BER of BPSK versus the average SNR of first path, $\bar{\gamma}_{1}$, for the Full GSC and the Block Change schemes over non-identical/correlated Rayleigh fading channels when $L=5, L_{c}=3, L_{a}=2$, and $\gamma_{T}=5$ $\mathrm{dB}$.

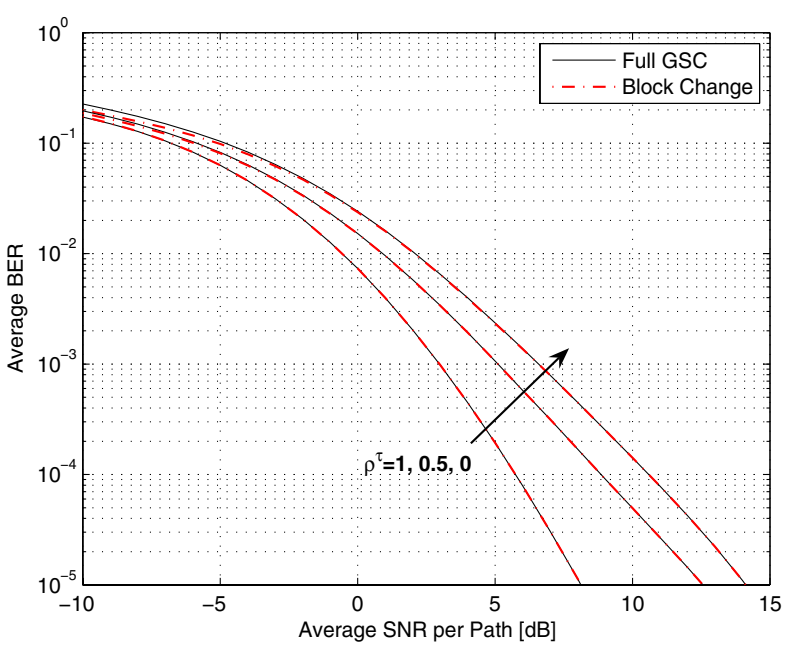

(a) $\gamma_{T}=-5 \mathrm{~dB}$

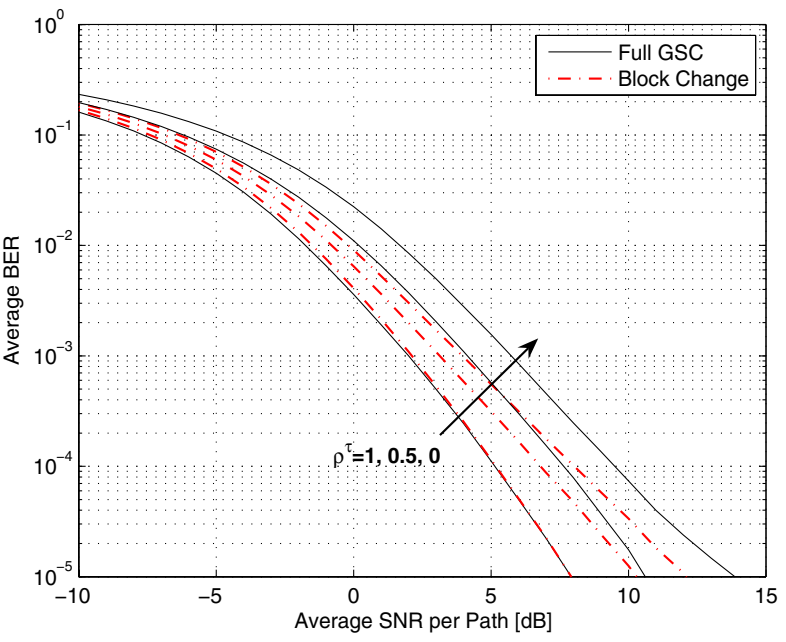

(b) $\gamma_{T}=5 \mathrm{~dB}$

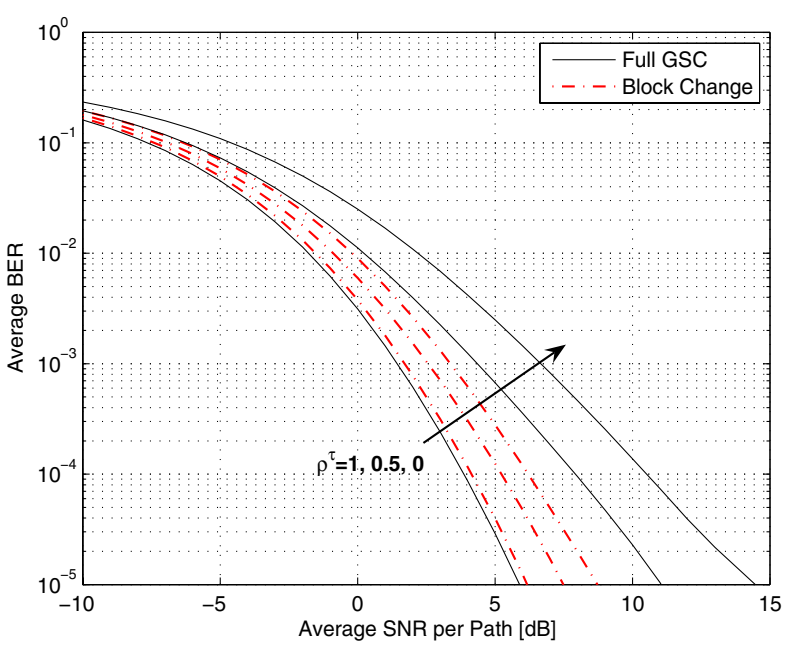

(c) $\gamma_{T}=15 \mathrm{~dB}$

Fig. 3. Average BER of BPSK versus the average SNR per path, $\bar{\gamma}$, for the Full GSC and the Block Change schemes with outdated channel estimation over i.i.d. Rayleigh fading channels when $L=5, L_{c}=3$, and $L_{a}=2$. 